\title{
Die Erinnerung an Vergessenes in der Byzantinistik seit ihren Anfängen im 15./16. Jahrhundert
}

\author{
FRANZ TINNEFELD (München)
}

Der Tübinger Gräzist Martin Crusius leitete um 1600 seine Oratio in Pulcheriam Augustam, eine oströmische Kaiserin im 5. Jahrhundert, mit einer Betrachtung über die Aufgabe des Historikers ein, das Vergangene der Vergessenheit zu entreißen, und verglich ihn mit dem Plünderer eines Grabes (tymborychos), ${ }^{1}$ vielleicht ein zu negativ besetztes Bild für das durchaus sinnvolle Leitthema der drei Teilkonferenzen Erinnern und Vergessen in den Kulturen Südosteuropas, dem sich der vorliegende Beitrag einordnet. Allerdings geht es hier nicht um die Erinnerung an Vergessenes in einer Kultur Südosteuropas, sondern in der Wissenschaft, die sich mit Byzanz beschäftigt, einer Kultur, die territorial nur zum Teil in Südosteuropa angesiedelt war. Im folgenden gehe ich der Geschichte der Byzantinistik vom 16. bis zum 20. Jahrhundert ${ }^{2}$ nach und versuche ohne Anspruch auf Vollständigkeit die allmähliche Formung dieser Kulturwissenschaft aus dem andauernden Bemühen zu erweisen, vergessene Aspekte und Arbeitsfelder des Faches zu entdecken und wissenschaftlich $\mathrm{zu}$ durchdringen. Dabei gehe ich nach Möglichkeit, wenn auch nicht in jedem einzelnen Fall, der Frage nach, welche Umstände jeweils zu solchen Entdeckungen führten.

Ich verzichte auf eine Berücksichtigung der Byzanzrezeption in der Frühzeit des italienischen Humanismus, ${ }^{3}$ die noch an ein in Rudimenten fortdauerndes „Reich“ anknüpfen konnte. Es ist jedoch festzuhalten, dass in der Zeit um das Ende von Byzanz, also kurz vor und nach der Eroberung Konstantinopels durch die Türken im Jahr 1453, eine solche Rezeption erstmals möglich wurde, weil zunächst in Italien und in anderen Ländern Westeuropas das humanistische Interesse an der Antike den Import einer großen Zahl griechischer Handschriften begünstigte. Diese enthielten auch mancherlei Texte aus byzantinischer Zeit, die allerdings zunächst kaum Beachtung fanden. Der Zugang zu dieser Literatur wurde den Humanisten aber wenigstens durch eine ihr eigene Besonderheit erleichtert: Sie ist zu einem hohen Prozentsatz in einem Griechisch verfasst, das von der antiken Tradition geprägt ist und den Humanisten

1 Suchland 2001, S. 101.

2 Knappe Überblicke geben Ostrogorsky 1963, S. 1-18; Moravcsik 1976, S. 18-54; Mazal 1989, S. 13-22. Zu Kurbatov 1975, s. u., Anm. 38.

3 Zum Thema „Byzantinistik in der Humanistenzeit“ vgl. Pertusi 1966, S. 3-25. 
daher mühelos zugänglich war. So kam es allmählich zu einer Byzanzrezeption größeren Umfanges.

Am Anfang steht in Deutschland der Name des Augsburger Humanisten Hieronymus Wolf (1516-80). ${ }^{4}$ Er war nach eigenem Bekunden ein begeisterter Philologe der antiken Klassik ${ }^{5}$ und hatte in jungen Jahren die attischen Redner Isokrates und Demosthenes ins Lateinische übersetzt. ${ }^{6}$ Damit als Kenner der alten Sprachen ausgewiesen, ${ }^{7}$ wurde er 1551 Sekretär und Bibliothekar des humanistisch gesinnten Kaufmannes Hans Jakob Fugger, eines Neffen des Firmenchefs Anton Fugger. ${ }^{8}$ Wolf gebührt die Ehre, erstmals byzantinische Historikertexte für eine Druckausgabe ediert und ins Lateinische übersetzt zu haben. Er hätte jedoch die verachteten byzantinischen Autoren wohl kaum ohne einen Anstoß von außen zur Kenntnis genommen. Diesen gab der aus Böhmen stammende Humanist Hans Dernschwam (1494-1568/69), ${ }^{9}$ der bis 1549 als Faktor der Augsburger Fugger gearbeitet hatte ${ }^{10}$ und im Jahr 1553 als Privatmann, aber im Auftrag Anton Fuggers, eine kaiserliche Gesandtschaft ${ }^{11}$ nach Konstantinopel und Anatolien begleitete, um dort griechische Handschriften zu erwerben. Im Jahr 1555 kam er mit reicher Ausbeute zurück, stolz darauf, auch einige Manuskripte byzantinischer Historiker erworben zu haben, darunter einen vollständigen Kodex und zwei Teilhandschriften der byzantinischen Chronik des Johannes Zonaras, die mit der biblischen Weltschöpfung und der Geschichte des Volkes Israel beginnt und dann die Zeit des römischen und oströmischen Reiches bis zum frühen 12. Jahrhundert behandelt, ${ }^{12}$ sowie

4 Zu Wolf siehe u. a. Beck 1984; Zäh 1992; Reinsch 1994, S. 53-57.

5 Beck 1984, S. 16, 46, 49.

6 Ebd., S. 59.

7 Wamser 2004, S. 426 (Helmut Zäh).

8 Es war nach dem Tode Anton Fuggers (1493-1560) Hans Jakob Fugger, der 1571 dem Bayernherzog Albrecht V. die Fuggerbibliothek übereignete, zur Deckung der reichlichen Finanzhilfe, die der Herzog dem verschuldeten Hause Fugger geleistet hatte.

9 Zu Dernschwam siehe Reddig 1990.

10 Er war zuständig für die Organisation der Kupferproduktion in Neusohl (heute Banská Bystrica, Slowakei) und für andere Wirtschaftsunternehmen der Fugger.

11 Es handelte sich um eine Gesandtschaft, die von Kaiser Ferdinand I. nach Konstantinopel entboten worden war und von Franz Zay, einem ungarischen Magnaten und Admiral der Donauflotte, und von Anton Verantius, Bischof von Fünfkirchen, geleitet wurde (Reddig 1990, S. 47-62).

12 Gemäß Büttner-Wobst 1892, S. 202-217, benutzte Wolf für seine Zonaras-Edition fünf Handschriften. Von diesen hatte Dernschwam in Konstantinopel bzw. in Pera/Galata gekauft: 1. Monac. gr. 324 (mit dem gesamten Text der Chronik, gegliedert in Geschichte Israels von der Weltschöpfung bis zur Zerstörung Jerusalems $70 \mathrm{n}$. Chr. und Geschichte des römisch-byzantinischen Reiches bis zum Jahr 1118); 2. Monac. gr. 325 (enthält die Chronik ab Buch 10, also mit dem Beginn der römischen Geschichte); 3. Eine heute verschollene Handschrift, die Dernschwam ex vetusto codice hatte abschreiben lassen (nur Bücher 13 und 14, von Konstantin I. bis zu Justinian I.). Außerdem benutzte Wolf noch zwei weitere Handschriften: 4. Monac. gr. 93 (Bücher 13-18 
eine Handschrift mit dem Geschichtswerk des Niketas Choniates, das dem 12. und frühen 13. Jahrhundert gewidmet ist. ${ }^{13}$ Dernschwam wies Anton Fugger auf die Bedeutung dieser Handschriften für die Erforschung des oströmischen Reiches hin und konnte ihn dafür gewinnen, Wolf mit der Edition und lateinischen Übersetzung beider Werke zu beauftragen. Zusammen mit einem Gehilfen machte sich Wolf, dem Fugger reichlichen Lohn in Aussicht stellte, ${ }^{14}$ an die Arbeit, für die er noch weitere Handschriften heranzog, und konnte sie innerhalb von zwei Jahren abschließen. Im Jahr 1557 erschienen Zonaras und Choniates in zwei getrennten Bänden beim Oporinus-Verlag Basel im Druck. ${ }^{15}$ Als nun Anton Fugger auch noch eine Edition und Übersetzung der Rhomäischen Geschichte (Rhomaïké Historía) des Nikephoros Gregoras, von der in der Fuggerbibliothek nur eine fragmentarische Handschrift vorlag, ${ }^{16}$ von ihm verlangte, war Wolf, seit 1557 Rektor des St.-Anna-Gymnasiums in Augsburg, nicht gerade begeistert, erledigte aber auch diese Aufgabe in kurzer Zeit, vor allem, um seinen Herrn nicht zu verärgern. ${ }^{17}$ Dennoch erschien der GregorasBand, ergänzt durch eine bereits vorliegende lateinische Übersetzung des Laonikos Chalkokondyles, erst im Jahr 1562, wiederum bei Oporinus. ${ }^{18}$ Das gesamte Übersetzungswerk wird im Titel dieser Ausgabe zutreffend als Integrum

zur byzantinischen Geschichte; zum Text des Niketas Choniates in dieser Handschrift s. u., Anm. 13); 5. Die Handschrift Vindob. hist. gr. 16, die 1513 aus Buda nach Wien gelangt war und von bzw. für Wolf entliehen wurde.

13 Von Niketas Choniates hatte Dernschwam einen Kodex mitgebracht, der sich heute noch im Besitz des Fuggerarchivs befindet (V. 159 Vol. A). Er erwarb sie wie Monac. gr. 325 von Alexandros Chartophylax, der sie, wohl für ihn, kopierte. Zu diesem Fuggeranus siehe van Dieten 1975, hier Bd. I, S. LII. Wolf benutzte außerdem die heutigen Handschriften Monac. gr. 93 der Fuggerbibliothek (vgl. zu dieser oben, Anm. 12, und van Dieten, ebd., S. CV und L) und Monac. gr. 450 aus der Augsburger Stadtbibliothek (van Dieten, ebd., S. XXXIII). Von diesen dreien enthält Monac. gr. 450 die sog. vulgärgriechische Paraphrase des Werkes, besser als ,umgangssprachliche Metaphrase“ zu bezeichnen, welche die Fugger 1544 von Antonios Eparchos erwarben. Wolf hielt diese Metaphrase für die von Niketas stammende Originalform, abgefasst ,im Dialekt der damaligen Zeit“, und den in den beiden anderen Handschriften überlieferten Originaltext des Niketas für eine spätere Paraphrase, die aber seiner Kenntnis des klassischen Griechisch eher entsprach. Vgl. Reinsch 1994, S. 54 f.

14 Beck 1984, S. 77f.; Zäh 1992, S. 93 (Text), S. 240 (Kommentar); Wamser 2004, S. 430, Nr. 992 (Helmut Zäh). Zur Bezahlung (372 Gulden für Wolf): Beck 1984, S. 89; Zäh 1992, S. 103. Über die Verzögerung der in Aussicht gestellten Zahlungen durch den Basler Oporinus-Verlag siehe Husner 1949, S. 150-156.

15 Zäh 1992, S. 316-318; Wamser 2004, S. 430, Nr. 992 (Helmut Zäh).

16 Es handelte sich um die Handschrift Monac. gr. 153, die von den 37 Büchern des Werkes nur die Bücher 1-11 (bis zum Jahr 1341) umfasste. Siehe van Dieten 1975a, S. 52-54; Zäh 1992, S. 261 (Kommentar); Reinsch 1994, S. 56.

17 So die Autobiographie (Beck 1984, S. 95; Zäh 1992, S. 109f.).

18 Zäh 1992, 322. Wamser 2004, S. 430, Nr. 993 (Helmut Zäh) zeigt und bespricht ein in München aufbewahrtes Exemplar, in dem die Choniates-Edition von 1557 mitgebunden ist. 
Byzantinae historiae corpus a Constantino magno ad Constantinum postremum bezeichnet; ${ }^{19}$ denn die Chronik des Zonaras deckte die Zeit bis zum frühen 12. Jahrhundert ab, Niketas Choniates das 12. und frühe 13., Gregoras das 13. und das 14. Jahrhundert bis 1341 und Chalkokondyles das spätere 14. und das frühere 15. Jahrhundert.

Man hat Wolf den „Vater der deutschen Byzantinistik“ genannt, doch ist abschließend noch einmal zu betonen, dass er zeitlebens ein Verehrer der Antike blieb und wohl kaum je ein ,,väterliches“ Empfinden für seine byzantinischen Autoren aufbrachte. ${ }^{20}$ Ich habe die Entstehung dieser Historikerausgaben wegen ihrer Bedeutung etwas ausführlicher geschildert, aber auch, um Wolfs „Vaterschaft wider Willen“ deutlich werden zu lassen. Dennoch darf man es als sein besonders Verdienst hervorheben, durch seine bahnbrechende Leistung die Konzentrierung des Humanismus auf die Antike überwunden und bereits im ersten Anlauf die gesamte Geschichte von Byzanz als Gegenstand der Forschung in Erinnerung gebracht zu haben.

Zeitlich der nächste bedeutende Humanist, der ein neues Arbeitsfeld der Byzantinistik erschloss, war Johannes Löwenklau ${ }^{21}$ (Leunclavius), geboren 1541 in Coesfeld/Westfalen, gestorben 1594 in Wien. Philologische Studien betrieb er u.a. bei Philipp Melanchthon und Wilhelm Holzmann (Xylander). Er ist als Rechtshistoriker bekannt, aber er begann mit Übersetzungen griechischer Historiker ins Lateinische, unter denen die Byzantiner Zosimos, Michael Glykas und Konstantinos Manasses, aber auch ein von Kaiser Manuel II. verfasster Fürstenspiegel zu nennen sind. ${ }^{22}$ Er war nicht der einzige, der in der Zeit des Humanismus byzantinische Rechtsquellen übersetzte, ${ }^{23}$ denn diese waren bereits seit dem 15. Jahrhundert mehr und mehr gefragt, weil sie zum besseren Verständnis bzw. zur Textemendation des im Westen geltenden Corpus Iuris Civilis Kaiser Justinians hilfreich waren. ${ }^{24}$ Aber es ist das Verdienst Löwenklaus, byzantinische Rechtsquellen in großem Umfang erschlossen und damit die byzantinische Rechtsgeschichte als Forschungsgegenstand entdeckt zu haben. Seine Übersetzung der Collectio Tripartita, einer im 6. Jahrhundert verfassten Sammlung kirchenrechtlich relevanter Fragmente aus dem Corpus

19 Zäh 1992, S. 322.

20 Beck 1984, S. 155: „Es war nicht sein eigener Wunsch und erst recht nicht seine eigene Entdeckung, wenn er sich daran machte, eine Art Corpus der byzantinischen Historiographie zu schaffen.“ Reinsch 1994, S. 54: „Wolf ist zunächst nur ausführendes Organ, das Objekt ist wenig geliebt." Darauf zeigt Reinsch vor allem, wie wenig Wolf die Zonaras-Chronik zusagte.

21 Der Name hat mit einer Löwenklaue nichts zu tun, sondern ist aus Ludolfing + Lohe (= Wald) zusammengesetzt (Fögen 1994, S. 197-199).

22 Reinsch 1994, S. 61; vgl. Fögen 1994, S. 199, die auch griechische Kirchenvätertexte nennt.

23 Siehe Osler 1994, S. 202-204.

24 Ebd., S. 202. 
Iuris Civilis, ist aus der Sicht des praktischen Bedürfnisses als sein wichtigster Beitrag zu diesem Arbeitsbereich zu nennen. ${ }^{25}$

War es also bei Löwenklau mehr noch als die unleugbare philologische Begeisterung die Notwendigkeit, die ihn bewog, die Quellen des abendländischen Rechtes zu erschließen, so darf man bei Martin Crusius (= Kraus) (1526-1607) das persönliche Interesse an seinem Gegenstand noch stärker im Vordergrund sehen. Allerdings kann man ihn kaum als Humanisten, sondern eher als Philhellenen bezeichnen, ,zu einer Zeit, als die Deutschen und die anderen Mitteleuropäer zwar sehr gut Bescheid wussten über die alten Hellenen, von den Bewohnern des türkisch besetzten Griechenlands aber recht unklare Vorstellungen hatten “. ${ }^{26}$ Crusius war als erster bereit, eine Entwicklung der griechischen Sprache anzuerkennen, die von der Klassik wegführte und in Byzanz deutliche Spuren hinterlassen hatte. Was Crusius also eigentlich vor dem wissenschaftlichen Vergessen rettete, war die lebendige Sprache der Griechen. ${ }^{27}$ Aber noch ein anderer Aspekt seines Lebenswerkes ist zu würdigen, der mit dem ersten zusammenhängt, die Entdeckung des „Byzance après Byzance “28, also des Fortlebens der byzantinischen Kultur nach dem Fall Konstantinopels. Diesem Aspekt sind seine beiden Hauptwerke, die Turcograecia (1584) und die Germanograecia (1585), gewidmet. Der Protestant Crusius schätzte die Griechen seiner Zeit aber auch als das Volk, welches zur Ursprache des Neuen Testamentes einen unmittelbaren Zugang hatte und daher mit den Reformatoren die besondere Wertschätzung der Bibel teilte. ${ }^{29}$

Den von Wolf eingeschlagenen Weg ging vor allem sein Schüler und späterer Nachfolger als Rektor am St.-Anna-Gymnasium zu Augsburg David Hoeschel (1556-1617) entschlossen weiter. Nicht weniger als 44 griechische und byzantinische Autoren wurden von ihm herausgegeben. Darunter sind vor allem solch bedeutende Werke zu nennen wie die Bibliothek des Photios (eine umfangreiche Sammlung von Lesefrüchten des gelehrten Patriarchen), die Geschichte der Kriege Justinians I. von Prokop und die Alexiás der Anna Komnene über die Taten ihres Vaters Alexios I. Die Alexiás lag ihm allerdings nur in einer Epitome vor, die er irrig für den ersten Teil des Originalwerkes hielt. Im Gegensatz zu Crusius hatte Hoeschel keine Kenntnis der griechischen Umgangssprache. Er scheint sich aber weit mehr als Wolf aus eigenem Antrieb

25 Stolte 1994, S. 219-221; Fögen 1994, S. 200.

26 Eideneier 1994a, S. 123.

27 Ebd., passim.

28 Diesen Titel trägt ein 1935 in Bukarest erschienenes Buch des bedeutenden rumänischen Historikers und Byzantinisten Nicolae Jorga.

$29 \mathrm{Zu}$ diesem protestantischen Hintergrund des Philhellenismus bei Crusius siehe Wendebourg 1994, S. 119f. Sie zeigt, dass sich bei Crusius die Liebe zu allem Griechischen mit der „Liebe zu dem von der Reformation in seiner Reinheit wiederentdeckten Evangelium “ verband. Er verstand das Griechische als die Sprache des Neuen Testamentes und ,jenes Zweiges der Christenheit, der die evangelische Wahrheit besser bewahrt hat als der lateinische". 
mit den byzantinischen Autoren beschäftigt zu haben und zeigt auch mehr historisches Verständnis als sein Vorgänger. Sein bleibendes Verdienst ist es, bedeutende Geschichtsschreiber bereits früh vor dem Vergessen bewahrt zu haben. ${ }^{30}$

Im 16. Jahrhundert entdeckte man vor allem die griechische Hagiographie, Patristik und Konzilienkunde, zunächst in Italien, etwas später auch in Deutschland, hier an der Jesuitenuniversität in Ingolstadt. Doch verlagerte sich der Schwerpunkt der theologischen Byzantinistik bereits gegen Ende des 16. Jahrhunderts nach Frankreich ${ }^{31}$, das im 17. Jahrhundert eine führende Rolle auch für andere Teilbereiche des Faches spielen sollte. So war auch der bedeutendste Byzantinist des Jahrhunderts, Charles Dufresne, sieur Du Cange (1610-88), ${ }^{32}$ kein Theologe. Er arbeitete zunächst auf den Gebieten Genealogie und Heraldik und wandte erst im Alter von 45 Jahren seine Aufmerksamkeit dem byzantinischen Osten zu, als er eine Neuedition mit französischer Übersetzung des Historikers Geoffroy de Villehardouin vorbereitete. Villehardouin hatte im frühen 13. Jahrhundert den fatalen Vierten Kreuzzug und die Eroberung Konstantinopels durch die Lateiner 1204 beschrieben. Auf die Edition ließ Du Cange 1657 eine Histoire de l'empire de Constantinople sous les empereurs français, also eine Geschichte des Lateinischen Kaiserreiches, folgen, die erstmals den besagten Einbruch abendländischer Mächte in das byzantinische Herrschaftsgebiet gebildeten Kreisen im Westen bekanntmachte. Allerdings war $\mathrm{Du}$ Cange weit davon entfernt, diesen Vorgang wie die Byzantiner selbst und wie die meisten Historiker der Gegenwart eher negativ zu beurteilen. In einem an Ludwig XIV. gerichteten Einleitungsschreiben und im Vorwort zu diesem Werk vertritt er vielmehr den Standpunkt, Gott habe die Eroberung Konstantinopels im Jahr 1204 geschehen lassen, um die Griechen zu strafen und die Kirchenunion mit dem Osten durchzusetzen. Zugleich betont er mit Stolz und nicht ohne Spitze gegen das deutsche Kaisertum der Habsburger, dass die lateinischen Kaiser Franzosen waren (worauf bereits der Titel seines Werkes hinweist). Ja, er fordert den König von Frankreich gerade$\mathrm{zu}$ auf, das türkische Reich anzugreifen und das französische Kaisertum von Konstantinopel wiederherzustellen. Seine Beschäftigung mit Byzanz, so dankenswert sie ist, war also in hohem Grad vom Geist einer französischen Nationalpolitik bestimmt. Dies gilt auch für seine 1680 erschienene Historia Byzantina duplici commentario illustrata, ein geniales Werk, dessen erster Teil die Topographie Konstantinopels und dessen zweiter die Genealogie der byzantinischen Adelsfamilien behandelt, beides Aspekte der Byzantinistik, die bis dahin noch völlig unerforscht waren. Wie das Widmungsschreiben an Ludwig XIV. zeigt, ist auch die Historia Byzantina einer imperialen Idee verpflichtet,

30 Angaben über Hoeschel nach Reinsch 1994, S. 58f. Die erwähnte Handschrift mit der Epitome der Alexiás ist der heutige Codex Monac. gr. 355.

31 Beck 1959, S. 7-9.

32 Das Folgende nach Spieser 2000, S. 200. 
die Frankreich als direkten Nachfolger des römisch-byzantinischen Reiches versteht. Sie steht zugleich in Verbindung mit dem 1648 von Philippe Labbé, dem bedeutenden Erforscher der Konzilsakten, offiziell begründeten Pariser Corpus der byzantinischen Historiker, dessen Leitung Du Cange seit den siebziger Jahren übernahm. Bis zum Jahr 1711 erschienen insgesamt 34 Bände. Damit war nun die bisher nur teilweise bekannte byzantinische Geschichtsschreibung in vollem Umfang erschlossen.

Die letzte bedeutende Frucht seiner Byzanzstudien, das Glossarium ad scriptores mediae et infimae graecitatis, dem 1678 ein entsprechendes Werk für das Mittellatein vorausgegangen war, erschien kurz vor seinem Tod im Jahr $1688 .{ }^{33}$ Es war die erste Publikation überhaupt, die mit umfangreichem Material der bis dahin kaum beachteten Tatsache Rechnung trug, dass zahlreiche byzantinische Texte einen weit von der Klassik entfernten Wortschatz verwenden.

Es sei wenigstens kurz darauf hingewiesen, dass die 1618 gegründete französische Benediktinerkongregation des Hl. Maurus (Mauriner) bedeutende Gelehrte hervorbrachte, die auch für bis dahin „vergessene“ Wissenszweige der Byzantinistik den Grund legten. Hier sei vor allem der Zeitgenosse von Du Cange Jean Mabillon (1632-1707) genannt, der die Basis für eine allgemeine Urkundenlehre legte, aber auch Bernard de Montfaucon (1655-1741), der erste Verfasser einer Palaeographia graeca. Da die überwiegende Mehrzahl der griechischen Handschriften aus byzantinischer Zeit stammt, ist auch die Abfassung einer griechischen Paläographie ein Akt des Erinnerns, der die Byzantinistik betrifft.

Im 18. Jahrhundert traten wieder einige deutsche Byzantinisten in den Vordergrund, unter ihnen Johann Albert Fabricius, der mit seiner Bibliotheca graeca in 14 Bänden (1705-28) eine erste materialreiche Basis für eine byzantinische Literaturgeschichte legte, und Johann Jakob Reiske (1716-74), ${ }^{34}$ Professor der Klassischen Philologie in Leipzig. Im Jahr 1731 hatte die Leipziger Ratsbibliothek die einzige vollständige Handschrift des im 10. Jahrhundert kompilierten byzantinischen Zeremonienbuches, einer Quelle von einmaliger Bedeutung für das Ritual am Kaiserhof, erworben. Mit der Edition der Handschrift wurde zunächst Johann Heinrich Leich beauftragt, der aber in jungen Jahren starb. Eher zufällig und widerwillig (weil er das Griechisch dieser Textsammlung durchweg als „barbarisch“ verachtete) übernahm Reiske die Arbeit, da sie gut bezahlt wurde. Doch ist seine Edition, die 1751 mit lateinischer Übersetzung erschien, dennoch als genialer Wurf zu bewerten. Reiske verfasste auch einen bemerkenswerten Kommentar zur Edition, von dem aber 1754 zunächst nur der erste Teil erschien.

33 Siehe den Artikel Du Cange 1, in Baltaeu 1967, Sp. 1135f, in dem seine sämtlichen Werke aufgezählt sind.

34 Das Folgende nach Rochow 1976, S. 193-197. 
Weder in Deutschland noch in Frankreich war bislang eine große Synthese der byzantinischen Geschichte vorgelegt worden. Nun erschien eine solche fast gleichzeitig in Frankreich von Charles Lebeau in den Jahren 1767-86 und in England von Edward Gibbon 1776-88, und beide beurteilten die byzantinische Phase des Römischen Reiches als Verfallszeit. ${ }^{35}$ Der bedeutendere Historiker aber war Gibbon mit seinem sechsbändigen Werk The history of the decline and fall of the Roman Empire, vielleicht bis heute der bemerkenswerteste Versuch einer geschichtsphilosophischen Deutung des Phänomens Byzanz. ${ }^{36}$ Es scheint, dass Gibbon aus persönlichem Engagement und nicht aus sachfremden Motiven zur Abfassung seiner History gelangte. ${ }^{37}$ Es war das große Römische Reich, das Gibbon zugleich faszinierte und abstieß. Ruhmreich waren aus seiner Sicht nur die klassischen Anfänge, und was folgte, war ständiger Niedergang. Man hat gefragt, inwiefern hier der Verfall der britischen Macht zur Zeit der Emanzipation Amerikas eine Rolle spielte. Aber weder in seiner Korrespondenz noch in seinen sonstigen Äußerungen zeigt Gibbon an diesen Vorgängen Interesse. Weit stärker scheint der Einfluss der Kritik am Konzept der Universalmonarchie zu sein, die man seit der Zeit Karls V. zunächst durch die habsburgischen Kaiser verkörpert sah. Es war zuerst der Niederländer Hugo Grotius (1583-1645), der jegliche Reichsideologie ablehnte und an ihrer Stelle ein Konzept souveräner Nationalstaaten in Europa befürwortete, und er fand Nachfolger auch bei englischen Philosophen. Zu neuem Widerspruch forderten die Tendenzen Ludwigs XIV. heraus, eine französische Universalmonarchie zu begründen. Im 18. Jahrhundert waren es Pietro Giannone, Charles de Montesquieu, David Hume und William Robertson, die zur Diskussion um die Universalmonarchie beitrugen und Gibbon Argumente zu deren negativer Beurteilung lieferten. Sowohl Montesquieu wie Hume sahen in einer Fortsetzung der Universalmonarchie Ludwigs XIV. eine Bedrohung, und es war Montesquieu, der mit seinem Werk Considérations sur les causes de la grandeur des Romains et de leur décadence das unmittelbare Präludium zu Gibbons Werk und die entscheidende Anregung zu seiner Abfassung lieferte. Montesquieu zollte der römischen Republik uneingeschränkte Bewunderung und tadelte das Kaisertum als Tyrannei, aber der entscheidende Schritt zum Niedergang war aus seiner Sicht die Reichsteilung, an der er Konstantin dem Großen die Schuld gab. Es ist allerdings zu betonen, dass sich Gibbon keineswegs sklavisch an das Werk Montesquieus anlehnte, sondern vielmehr intensiv mit ihm auseinandersetzte. Der Gedanke, der Gibbon bei Abfassung seines Werkes vor allem leitete, war die Überzeugung, dass das römische Universalreich in byzantinischem Gewand zwar fortdauerte, zugleich aber in Einförmigkeit, Passivität und selbstgenügsamer Isolation erstarrte und als solches noch lange und allzu lange da-

35 Ostrogorsky 1963, S. 4.

36 So bezeichnet Easterling 2000, S. 119, Gibbons Werk als „,beyond all comparison the greatest literary production of the eighteenth century".

37 Die folgenden Gedanken entnehme ich aus Robertson 1997, S. 247-270. 
hinvegetierte. Damit glaubte er zugleich nachgewiesen zu haben, dass die Idee der Universalmonarchie auf die Dauer chancenlos sei. Er konnte die Kaiserkrönung Napoleons zehn Jahre nach seinem Tode noch nicht vorausahnen, aber gerade für dessen kurzlebiges Universalreich treffen ja die Bedenken Gibbons in vollem Maße zu.

Im 18. Jahrhundert sind auch die Anfänge einer wissenschaftlichen Byzantinistik in Osteuropa ${ }^{38}$ zu konstatieren. In Russland hatte bereits seit dem 16. Jahrhundert die Staatsideologie das Kaisertum und den (allerdings übertrieben hoch angesetzten) Zentralismus des byzantinischen Reiches als vorbildlich bewertet. Später entdeckte man die eigenen Anfänge in der Kiever Rus' und deren Kontakte zu Byzanz. 1764 veröffentlichte A.L. Schlözer (Šljocer) sein Werk über das alte Russland unter Verwendung griechischer Quellen und regte die Herausgabe einer vierbändigen Sammlung byzantinischer Quellentexte über die Kiever Rus' an. ${ }^{39}$ Im 19. Jahrhundert verdient vor allem die überragende Persönlichkeit des Byzantinisten Vasilij Grigor'evič Vasil'evskij (1838-99) Beachtung. Er erforschte als erster in größerem Umfang die Sozialund Rechtsgeschichte, vor allem die Agrargeschichte von Byzanz, ${ }^{40}$ lange Zeit bevor auch Vertreter der Annales-Schule in Frankreich sich diesen Fragen widmeten. ${ }^{41}$

Die wichtigsten Beiträge der Balkanslawen waren die Werke, in denen sie ihre eigenen Beziehungen zu Byzanz darstellten. In Bulgarien verfasste Paisij Chilandarski (1722-98) die erste bulgarische Geschichte. Bald folgten weitere Autoren, aber erst Sp. Palauzov (1818-72) begründete eine wissenschaftliche bulgarische Geschichtsschreibung. Unter Benutzung auch byzantinischer Quellen verfasste er Bücher über den Zaren Simeon (1852), die Anfänge der Aseniden (1858) und über die Geschichte des bulgarischen Patriarchats (1860). Als erster behandelte er auch die byzantinisch-bulgarischen Beziehungen. ${ }^{42}$ Die Edition der griechischen Inschriften in Bulgarien durch Veselin Beševliev im 20. Jahrhundert ist ein bahnbrechender Beitrag zur byzantinischen Epigraphik, ${ }^{43}$ die im Übrigen als Disziplin über wichtige Ansätze noch nicht hinausgekommen ist. ${ }^{44}$

38 Dazu liefert Kurbatov 1975 wertvolles Material. Allerdings behandelt er nur einen Teilaspekt, die Geschichtsschreibung, und seine marxistische Interpretation drängt sich stellenweise störend in den Vordergrund.

39 Kurbatov 1975, S. 44-51.

40 Zum Begründer der sozialgeschichtlichen Schule Vasil'evskij im 19. Jahrhundert siehe vor allem Litavrin 1994, S. 5-21.

41 Hier ist für die Byzantinistik vor allem Lemerle mit seiner „Esquisse pour une histoire agraire de Byzance zu nennen“, die erstmals 1958 in: Revue historique 219 und 220 publiziert wurde.

42 Kurbatov 1975, S. 68; Dujčev 1966, S. 74-78.

43 Beševliev 1964; ders. 1963.

44 Siehe den Artikel „Epigraphy“ in: Oxford Dictionary of Byzantium, S. 711-713. Der Verfasser Cyril Mango beginnt mit der lapidaren Feststellung: „A discipline of Byz. 
Für Serbien sei zuerst Jovan Raić (1726-1801), der „Vater der serbischen Geschichtsschreibung", genannt. Er verfasste in vier Bänden eine Geschichte der Bulgaren, Kroaten und Serben, also der Balkanslawen insgesamt, die erste in einer slawischen Sprache. Mit diesem Werk wurde der Einfluss der Romantik mit ihrer Tendenz zur Idealisierung der Vergangenheit, welche die vorausgehende serbische Historiographie geprägt hatte, überwunden. Die serbischbyzantinischen Beziehungen wurden aber erst von Vuk Karadžić (1787-1864) ausführlicher behandelt. ${ }^{45}$ Aber erst im Jahr 1893 wurde erstmals ein Professor auf einen serbischen Lehrstuhl berufen, der im Rahmen einer allgemeinen Geschichte des Mittelalters auch Byzanz berücksichtigte. Es war der in Belgrad und Paris geschulte Božidar Prokić (1859-1922), der aber später auch noch einige Zeit bei Karl Krumbacher ${ }^{46}$ in München studierte. Seit 1906 lehrte auch Dragutin Anastasijević (1877-1950), im engeren Sinne ein Schüler Krumbachers, an der neu gegründeten Universität Belgrad und zwar, entsprechend seinen Studien in München, über die dort etablierten Fächer Mittelgriechische Literatur und Griechische Paläographie. Ein dritter serbischer Schüler Krumbachers war der Mönch Filaret Granić (1883-1948), ein Spezialist für Kirchengeschichte und verwandte Themen. Von drei weiteren serbischen Historikern, die kürzere Zeit am Münchener Institut arbeiteten, war vor allem Nikola Radojčić (1882-1964) auch als Byzantinist von Bedeutung. ${ }^{47}$

Der angesehenste Vertreter der ungarischen Byzantinistik ist bis heute Gyula Moravcsik (1892-1972). Als innovativ sind vor allem sein magistrales Werk Byzantinoturcica ${ }^{48}$ und seine Forschungen über die byzantinischungarischen Beziehungen zu bewerten.

Vor allem die frühe neugriechische Geschichtsschreibung sah im byzantinischen Reich den Vorläufer des modernen griechischen Staates, so Konstantinos Paparrigopulos in seiner sechsbändigen Geschichte der griechischen Nation (1860-77). Die Geschichte der „Hellenen“ begann aus seiner Sicht im Zeitalter Justinians. Er verstand den Ikonoklasmus als einen mit der Aufklärung vergleichbaren Vorgang und die Ereignisse von 1204 als Katastrophe, an der allein die abendländischen Mächte Schuld trugen. ${ }^{49}$ So weit die kurzen Bemerkungen zu den unterschiedlichen Akzenten der „Erinnerung“ in der Byzantinistik Osteuropas, die nur einige Hauptlinien andeuten.

epigraphy does not yet exist." Siehe aber neuestens die Forschung von Andreas Rhoby, Wien.

45 Kurbatov 1975, S. 69; Dujćev 1966, S. 79-86.

46 Zu Krumbacher s. u., Text mit Anm. 50-52.

47 Mit den hier erwähnten serbischen Byzantinisten befasste sich ausführlicher Ljubomir Maksimović in einem Vortrag zum Thema „Karl Krumbachers serbische Schüler“, gehalten am 25.02.2005 an der Universität München. Ich danke Herrn Kollegen Maksimović für die Überlassung des Manuskriptes.

48 Moravcsik 1958.

49 Kurbatov 1975, S. 71. 
Der wichtigste Beitrag zur Ausbreitung der byzantinischen Studien im frühen 19. Jahrhundert war zweifellos die Quellensammlung des Corpus Scriptorum Historiae Byzantinae, nach seinem Erscheinungsort kurz „Bonner Corpus“ der byzantinischen Historiker genannt, begründet 1828 von Barthold Georg Niebuhr (1776-1831), der seit 1823 Professor in Bonn war. Das neue Corpus, dessen Bände Oktavformat haben, sollte das frühere unhandliche Pariser Corpus der byzantinischen Historiker im Quartformat ersetzen. Es war im Wesentlichen ein Nachdruck des Pariser Corpus, wurde aber auch durch Neueditionen ergänzt. Jedenfalls wurde der Zugang zu den Geschichtsquellen und damit das „Erinnern“ durch das handliche Format und das erweiterte Angebot an Texten seitdem wesentlich erleichtert.

Aber noch fehlten eine umfassende Fachbibliographie und eine Orientierung in der byzantinischen Literatur durch ein übersichtliches Handbuch. Beides wurde erst von Karl Krumbacher (1856-1909) in München gegen Ende des 19. Jahrhunderts erstellt. ${ }^{50}$ Karl Krumbacher berücksichtigte in seinem Handbuch auch erschöpfend die bis dahin immer noch vielfach als barbarisch abgelehnte Literatur in der Volkssprache und erwies sich zeit seines Lebens als ein Vorkämpfer des gewachsenen, lebendigen Idioms der zeitgenössischen Griechen, dem er zuungunsten der erstarrten, an das antike Griechisch angelehnten sogenannten Reinsprache vor allem im griechischen Sprachraum zu größerer Anerkennung verhelfen wollte. ${ }^{51}$ Er habilitierte sich 1884 in München als erster Deutscher für das neue Fach „,Mittel- und neugriechische Philologie“. Auf einer Forschungs- und Orientierungsreise durch Griechenland und die Westtürkei konnte er seine Kenntnis der Volkssprache vertiefen. ${ }^{52}$ Immer stand bei ihm das Interesse am griechischen Menschen seiner Zeit im Vordergrund, so auch bei seinem Einsatz für die Herausgabe der byzantinischen Urkunden als unentbehrlicher Quellen für das Alltagsleben und die Sozial- und Rechtsgeschichte der Byzantiner. Vor allem sein Verdienst ist die Begründung eines entsprechenden internationalen Projektes zur Herausgabe und Erforschung des gesamten erhaltenen byzantinischen Materials dieser Quellengattung, das er für die Bayerische Akademie der Wissenschaften der Association Internationale des Académies im Jahr 1901 vorlegte. Im Jahr 1907 beschloss die Bayerische Akademie die Einrichtung einer Dienststelle zur Herausgabe eines Corpus der byzantinischen Urkunden, die bis vor kurzem bestand. Auch andere Länder trugen bereits vor dem Ersten Weltkrieg zur Erforschung by-

50 Mit der Byzantinischen Zeitschrift, deren erster Band im Jahr 1892 erschien und die bis zur Gegenwart fortbesteht, begründete Krumbacher eine internationale Fachbibliographie der Byzantinistik. Es sei hier darauf hingewiesen, dass von 1929 bis 1994 die Prager Fachzeitschrift Byzantinoslavica eine zweite Bibliographie anbot, die in höherem Maße die Literatur in slawischen Sprachen berücksichtigte. Krumbachers Geschichte der byzantinischen Litteratur von Justinian bis zum Ende des oströmischen Reiches erschien in München 1891 in erster; 1897 in zweiter, erheblich erweiterter Auflage.

51 Vgl. Tinnefeld 2002, S. 294-312.

52 Krumbacher 1886. 
zantinischer Urkunden bei. Die internationale Zusammenarbeit wurde aber durch diesen Krieg vorübergehend gestört. Danach wurden in Deutschland und Frankreich größere Unternehmen zu Erforschung der Urkunden begründet. Nach Krumbachers frühem Tod 1909 war es in Deutschland zunächst Paul Marc, ${ }^{53}$ dann vor allem Franz Dölger (1891-1968), der durch die Bearbeitung der Regesten aller byzantinischer Kaiserurkunden ${ }^{54}$ und durch Editionen von Urkundenmaterial, vor allem vom Berg Athos, das Projekt entschlossen weiterführte. Nach intensiven Vorbereitungen seit dem Jahr 1919 unter Gabriel Millet erschienen in Paris seit den dreißiger Jahren in der Reihe „Archives de l'Athos“ Editionen der Urkunden aus den Archiven der Klöster auf dem Berg Athos. ${ }^{55}$ Sicher spielte bei den einschlägigen Aktivitäten beider Länder auch ein gewisser nationaler Wetteifer eine Rolle. Gegenwärtig trägt auch die österreichische Byzantinistik zur byzantinischen Urkundenforschung wesentlich bei. ${ }^{56}$

Ein wichtiger innovativer Impuls ging seit dem späten 19. Jahrhundert von der französischen Kongregation des Augustinerordens von der Himmelfahrt Marïa, den Assumptionisten, aus. ${ }^{57}$ Sie gründete im Jahr 1895 das Seminar Saint-Léon in Kadiköy, dem antiken Chalkedon, bei Istanbul ${ }^{58}$, das dem Ziel dienen sollte, die orthodoxen Kirchen, vor allem die Griechen und Bulgaren, für die römische Kirche zurückzugewinnen. $\mathrm{Zu}$ diesem Zweck wurden dort ortsansässige romtreue Priester durch ein Kollegium von Professoren in den orthodoxen Traditionen ausgebildet. Doch war dieses Kollegium von Anfang an auf hochqualifizierte wissenschaftliche Forschung bedacht. „C'est ainsi que les apôtres se firent erudits“, bemerkt der Pariser Assumptionist Albert Failler treffend zu dieser Entwicklung. Das Forschungsgebiet dieser Wissenschaftler war naturgemäß die Kirche von Byzanz. Sie konzentrierten ihre Aufmerksamkeit vor allem auf die kirchliche Geographie und auf die Erschließung der Patriarchatsakten von Konstantinopel durch ein breit angelegtes Regestenwerk, welches 1931/32 begonnen und 1991 erfolgreich abgeschlossen wurde. ${ }^{59}$ Für die Jahre 1315-1402 wurde auch das in Wien aufbewahrte Patriarchatsregister von Konstantinopel ausgewertet, eine unschätzbar wertvol-

53 Müller 2004, S. 308-314.

54 Dölger (Hrsg.) 1924-1965. In aktualisierter Neuauflage erschienen danach Teil 1/1. Halbband (2009), Teil 1/2. Halbband (2003) sowie Teil 2 (1995) und Teil 3 (1977).

55 Angaben nach Dölger / Karayannopulos 1968, S. 11-16.

56 An dieser Stelle sei das von Otto Kresten initiierte und geleitete Projekt einer Edition der Auslandsschreiben byzantinischer Kaiser nach dem Kanzleiprinzip (statt nach dem bisher praktizierten Archivprinzip) erwähnt. Siehe Kresten / Müller 1993/94, S. 402-404. Zur Edition der Patriarchatsurkunden s. u., Text mit Anm. 60.

57 Die folgenden Ausführungen folgen Failler 1995.

58 Es war benannt nach den Päpsten Leo I., dem Papst des Konzils von Chalkedon und dem zeitgenössischen Papst Leo XIII, einem Förderer der Einrichtung.

59 Grumel / Laurent / Darrouzès 1972-91 (einschließlich der verbesserten Neuauflagen, welche frühere Faszikel ersetzen). 
le Quellensammlung, die zum ersten Mal bereits im 19. Jahrhundert von Franz Miklosich und Joseph Müller ediert worden war und gegenwärtig in Wien in einer bislang etwa zur Hälfte erschienenen kritischen Edition mit deutscher Übersetzung neu herausgegeben wird. ${ }^{60}$ Zur kirchlichen Geographie publizierten Assumptionisten je einen Band über die Kirchen und Klöster von Konstantinopel, ${ }^{61}$ einen weiteren über die Kirchen und Klöster der großen monastischen Zentren des byzantinischen Reiches ${ }^{62}$ und einen dritten über die Bischofslisten (notitiae) des Patriarchats von Konstantinopel. ${ }^{63}$ Ein wichtiges Nebenprodukt dieser Forschungen war ein Band über die profanen Bauten Konstantinopels. ${ }^{64}$

Weil das Bonner Corpus überwiegend keine kritischen Editionen enthält, die den Anforderungen der modernen Textphilologie entsprechen, wurde in der zweiten Hälfte des 20. Jahrhunderts das internationale Corpus Fontium Historiae Byzantinae (CFHB) begründet, dessen erster Band im Jahr 1967 erschien. ${ }^{65}$ Neben der Herausgabe der Quellen ist aber auch die Methode ihrer kritischen Interpretation ein wichtiges und von Historikern manchmal zu wenig beachtetes Arbeitsfeld. Es war vor allem Paul Speck (1928-2003), der sein Lebenswerk diesem Forschungszweig widmete. Seine originelle Methode, die Quellen ,gegen den Strich“ zu lesen, wurde zwar nicht ohne Widerspruch hingenommen, gab aber der modernen byzantinistischen Geschichtsforschung zweifellos wichtige Anregungen. ${ }^{66}$

Relativ spät wurde die byzantinische Kunst als eigener Forschungszweig entdeckt. Goethe war nicht der erste, aber sicher der erste bedeutende Abendländer, der seine Aufmerksamkeit ostkirchlichen Ikonen zuwandte. Auf der ersten italienischen Reise besuchte er Anfang Januar 1787 in Rom einen Gottesdienst im griechischen Ritus, noch ohne den Bildschmuck der Kirche (Santa Maria in Cosmedin) zu erwähnen. Erst bei seinem zweiten Aufenthalt in Venedig (März bis Juni 1790) fielen ihm in der Kirche San Giorgio dei Greci „altgriechische Gemälde“ auf, wie er sie nannte. Wie tief seine Eindrücke damals waren, zeigt eine drei Jahrzehnte später darüber verfasste Schrift, in der er unter anderem die intensivere Bilderverehrung der Griechen beobachtet. Die Ikonen, die Goethe in Venedig sah, sind zwar durchweg nachbyzantinisch;

60 Zur Bewertung und zum Fortgang dieser Edition siehe meine Rezensionen der bisher erschienenen drei Bände in: Byzantinoslavica 45(1984), S. 224-226; 47(1996), S. 399-401; Byzantinische Zeitschrift 95(2002), S. 698-700.

61 Janin ${ }^{2} 1969$.

62 Janin 1975.

63 Darrouzès 1981.

64 Janin ${ }^{2} 1964$.

65 Neuester Überblick über alle seit 1967 erschienenen Bände in: Jahrbuch der Österreichischen Byzantinistik 52(2002), S. 433-436. Danach erschienene und/oder in Vorbereitung befindliche CFHB-Editionen sind in den weiteren Bänden des Jahrbuches angezeigt.

66 Vgl. das Werkverzeichnis von Paul Speck in: Sode / Takács 2001, S. XI-XIX. 
doch gelten die hier beobachteten Besonderheiten wegen des traditionellen Charakters der Ikonenkunst auch für die byzantinische Epoche. ${ }^{67}$ Goethes weitgesteckte kunstgeschichtliche Interessen wurden vor allem durch seine seit 1810 gepflegten Kontakte mit dem bedeutenden Kunsthändler Sulpiz Boisserée gefördert. Außer in Venedig stieß er auch hier und da in Deutschland auf Werke der byzantinisch beeinflussten Kunst, und bald folgten auch gezielte Ankäufe solcher Objekte für das Großherzogliche Kunstkabinett zu Weimar, darunter vor allem Erzeugnisse der russischen Kunst. Goethe vertrat zwar, wie er in seiner Schrift Kunst und Altertum zeigt, eher einen kritischen Standpunkt gegenüber der byzantinischen Kunst, war aber bereit, seine Meinung aufgrund besserer Kenntnis des Materials zu korrigieren. ${ }^{68}$ Sein Versuch im Jahr 1814, in Russland mehr über die Ikonenproduktion in einer sog. „Priesterfabrik“ in Suzdal' zu erfahren, blieb jedoch leider erfolglos. ${ }^{69}$ Von Goethe bis zu den Anfängen einer wissenschaftlichen Erforschung der byzantinischen Kunst sollten noch etwa 70 Jahre vergehen. ${ }^{70}$

In diesem Zusammenhang seien auch die spätantike und die byzantinische Archäologie erwähnt, die zunächst nur im Schatten der klassischen Archäologie betrieben wurden. Erst im 20. Jahrhundert entwickelte sich das Fach „Spätantike (bzw. Christliche) Archäologie“ allmählich zu einem eigenständigen Fach. In jüngster Zeit sind auch die Anfänge eines selbständigen Faches „Byzantinische Archäologie“ zu konstatieren. ${ }^{71}$ Obwohl aber die Erkenntnis, dass die Archäologie der historischen und kulturhistorischen Forschung wichtige Ergebnisse liefert, seit langem bekannt ist, werden diese von der Geschichtsforschung immer noch zu wenig berücksichtigt. ${ }^{72}$

Ich beende diesen Beitrag mit einem Hinweis auf die innovativen Beiträge zur Byzantinistik in einigen wichtigen Forschungszentren der Gegenwart. Das Studium der lange Zeit wenig beachteten Philosophie der Byzantiner wurde durch die Arbeiten und die Herausgebertätigkeit von Linos Benakis an der Akademie Athen entscheidend gefördert. ${ }^{73}$

67 Effenberger 1990, S. 30f.

68 Ebd., S. 18-27.

69 Ebd., S. 8-12.

70 Siehe Der neue Pauly, Bd. 13, s. v. Byzantinistik, D: Kunstgeschichte, Sp. 587-592 (Arne Effenberger), hier Sp. 588: „Die eigentliche wiss. Behandlung der byz. Kunst setzte (...) Ende des 19. Jahrhunderts und Beginn des 20. Jahrhunderts (...) ein.“

71 So jedenfalls der Artikel „Archaeology“ in: Oxford Dictionary of Byzantium, S. 152f. Zitat: „Byz. archaeology is a relatively young field of scholarship (...) Byz. archaeology does not really exist as a discipline of its own, and - although there are significant exceptions (...) - most Byz. sites are explored in connection with the investigation of classical monuments."

72 So Rutgers 1998, S. 9f.

73 Vgl. Benakis 2002. Übersicht über alle seine Publikationen ebd., S. 707-717. Er ist Herausgeber der Reihe „Philosophi Byzantini“, von der in den Jahren 1984-2005 insgesamt 11 Bände erschienen sind. 
An der Universität Wien erhielt als erster Herbert Hunger (1914-2000) ${ }^{74}$ im Jahr 1962 einen Lehrstuhl für das Fach Byzantinistik. Zuvor hatte er mit der Erstellung eines Katalogs der ca. 1100 in der Österreichischen Nationalbibliothek aufbewahrten griechischen Handschriften begonnen, den er im Jahr 1994 vollenden konnte. Hunger etablierte am Wiener Institut bald ein angesehenes Forschungszentrum. Die wichtigste Schwerpunkte der dortigen byzantinistischen Arbeit sind: ${ }^{75}$ die schon erwähnte Edition des Patriarchatsregisters; ein groß angelegtes, noch nicht abgeschlossenes Unternehmen zur Erfassung der gesamten Geographie des byzantinischen Reiches, die Tabula Imperii Byzantini (TIB); ein Repertorium der griechischen Kopisten; ${ }^{76}$ ein Projekt „Alltag und materielle Kultur in Byzanz" ${ }^{\text {"77 }}$ und das 1996 abgeschlossene Prosopographische Lexikon der Palaiologenzeit (PLP). ${ }^{78}$ In diesem Zusammenhang sei auf weitere prosopographische Unternehmen anderenorts hingewiesen, die Prosopographie der mittelbyzantinischen Zeit (PmbZ) an der Berlin-Brandenburgischen Akademie der Wissenschaften, die mit dem Jahr 641 an das inzwischen abgeschlossene Londoner Unternehmen Prosopography of the Later Roman Empire (PLRE) anknüpft und bis 1025 reichen soll, während in London die byzantinische Prosopographie nunmehr von 1025 bis 1261 als Prosopography of the Byzantine Empire (PBE) weitergeführt wird. ${ }^{79}$ Die restliche Zeit bis 1453 wurde durch das Unternehmen PLP bereits abgedeckt. Ebenfalls der Wiener Schule zuzuordnen ist auch ein im Erscheinen begriffenes weiteres Desiderat der Forschung, ein umfassendes Lexikon der byzantinischen Gräzität. ${ }^{80}$

Eine besondere Entstehungsgeschichte unter den Forschungszentren des Faches hat die Institution „Dumbarton Oaks“ in Washington/USA. ${ }^{81}$ Ihre Existenz ist den weitgefächerten kulturellen Interessen und dem kompetenten Sammeleifer des US-Botschafters Robert Woods Bliss und seiner Gattin Mildred zu verdanken. Es waren drei weit auseinanderliegende Fachgebiete, für die sich das Ehepaar interessierte. Eines von diesen war die besonders von Mrs. Bliss geförderte byzantinische Kultur. In einem 1920 gekauften schlossähnli-

74 Die folgenden Angaben nach Kresten 2000.

75 Vgl. Koder 2000, S. VII.

76 Gamillscheg / Harlfinger 1981-1997 (und, ab Teil 3, 1997, Eleuteri) (Teil 1: Großbritannien, T. 2: Frankreich, T. 3: Rom mit dem Vatikan).

77 Dieser Themenbereich war allerdings zu Beginn des Wiener Projekts bereits nicht mehr ,vergessen“, denn es stand schon das sechsbändige Werk von Kukules 1948-55 zur Verfuigung, das für viele Fragen dieser Thematik eine erste solide Basis liefert. Doch stellt die Realienkunde nach Kukules neue methodische Ansätze bereit und verdient insofern eine Bewertung als innovativ.

78 Trapp et al. 1976-1994; Addenda zu Faszikel 1-12, 1995, Abkürzungsverzeichnis und Gesamtregister. 1996.

79 Angaben nach Lilie et al. 1998, S. 2-5.

80 Trapp et al. ab 1994. Bis 2007 sind sechs Faszikel bis zum Wort „prospelagízo“ erschienen.

81 Die folgenden Angaben gemäß Anastos 1992. 
chen Gebäude im geschichtsträchtigen Stadtteil Georgetown der USHauptstadt wurde eine hochqualifizierte Sammlung byzantinischer Kunstobjekte zusammengetragen und eine reich dotierte Fachbibliothek von über 100.000 Bänden aufgebaut. Der gesamte Bestand wurde bereits im Jahr 1940 vom Ehepaar Bliss der Harvard University in Cambridge (Massachusetts) urkundlich zur Verwaltung übertragen und zum Aufbau einer akademischen Einrichtung bestimmt. Seitdem erhielten Byzantinisten aus der internationalen Fachwelt, darunter auch viele Kunsthistoriker, Gelegenheit, als Stipendiaten oder in leitender Position an diesem einmaligen Studienzentrum zu arbeiten. In langjähriger Arbeit wurden hier auch die reichhaltigen Münz- und Siegelsammlungen des Hauses katalogisiert. ${ }^{82}$ Unter der Leitung des aus der Sowjetunion emigrierten Byzantinisten Alexander Kazhdan (1922-1997) entstand hier unter Mitarbeit von Alice-Mary Talbot und zahlreichen Fachkollegen das erste große Nachschlagewerk des Faches, das dreibändige Oxford Dictionary of Byzantium (ODB, 1991). Kazhdan begründete und leitete bis zu seinem Tod auch das sog. Hagiography Database Project, das entsprechend einem besonderen Interessenbereich Kazhdans der realienkundlichen Auswertung der hagiographischen Quellen mit Hilfe der Computertechnik dient. In Dumbarton Oaks wurde schließlich auch ein weiteres dringendes Desiderat der Byzantinistik konzipiert, eine groß angelegte Synthese der Wirtschaftsgeschichte, die inzwischen erschienene dreibändige Economic History of Byzantium ${ }^{83}$ mit 51 Beiträgen namhafter Experten, herausgegeben von der Harvard-Professorin und ehemaligen Direktorin von Dumbarton Oaks Angeliki Laiou (1941-2008).

Die zahlreichen Facetten einer Kulturwissenschaft wie der Byzantinistik werden nicht „an einem Tage“ entdeckt. Trotz der Unvollständigkeit meiner Ausführungen hoffe ich gezeigt zu haben, wie im Laufe der Jahrhunderte ein breit gefächertes Gesamtbild von Byzanz aus den verschiedensten mehr oder weniger ,vergessenen“, aber früher oder später entdeckten Teilaspekten Gestalt annahm. Im 16. Jahrhundert veranlassten eher äußere Umstände als wissenschaftliches Interesse Hieronymus Wolf, durch Edition und lateinische Übersetzung einschlägiger Geschichtsquellen einen ersten Überblick über den Verlauf der byzantinischen Geschichte zu geben. Johannes Löwenklau erschloss vor allem wichtige Quellen des byzantinischen Rechtes. Martin Crusius entdeckte das gesprochene Idiom der mittelalterlichen und neuzeitlichen Griechen und die Fortdauer der byzantinischen Kultur nach dem Untergang des byzantinischen Reiches. David Hoeschel erschloss nach Wolf die Texte weiterer byzantinischer Autoren. Im 17. Jahrhundert suchte und fand Du Cange

82 Obwohl sowohl Numismatik wie auch Sigillographie vorher keine „vergessenen“ Gebiete der Byzantinistik waren, dürfen die numismatischen Forschungen von Philip Grierson (1910-2006) sowie sigillographischen Arbeiten von Nikolaos Oikonomides (1934-2000) und John Nesbitt in Dumbarton Oaks als bahnbrechend bezeichnet werden.

83 Laiou 2002. 
den Ruhm der Franzosen im Vierten Kreuzzug und im Lateinischen Kaiserreich von Konstantinopel. Er wurde aber auch der Begründer einer Stadtgeographie des byzantinischen Konstantinopel und einer Genealogie und Prosopographie wichtiger byzantinischer Aristokratenfamilien. Ferner führte er das von Philipp Labbé begründete Pariser Corpus der byzantinischen Historiker fort, die erste systematisch geplante Reihe mit Editionen der byzantinischen Historiographen. Schließlich erschloss er durch ein umfangreiches Lexikon das von Crusius entdeckte mittelgriechische Idiom. Die lange Geschichte der byzantinischen Urkundenforschung gründet methodisch auf dem Werk Jean Mabillons, die griechische Paläographie wurde von Bernard de Montfaucon begründet, dessen Lebenszeit bereits weit in das 18. Jahrhundert hineinragt. Johann Albert Fabricius legte den Grund für die byzantinische Literaturforschung. Johann Jakob Reiske edierte und kommentierte die höchst wertvolle byzantinische Quellensammlung, die unter dem Namen „Zeremonienbuch “ bekannt ist. Den frühesten und vielleicht bedeutendsten Versuch eines Gesamtkonzeptes der byzantinischen Geschichte verdanken wir im späteren 18. Jahrhundert dem Engländer Edward Gibbon, mag sein Urteil über Byzanz auch entsprechend einer der Universalmonarchie feindlichen Zeitströmung eher negativ sein. In Russland hingegen sah man bereits seit dem 16. Jahrhundert im Zentralismus des byzantinischen Reiches ein Vorbild für das eigene Herrschaftssystem. Im 18. Jahrhundert entdeckte man hier die enge Verbindung zwischen den Anfängen der eigenen Geschichte und der byzantinischen Kultur. Vasilij Vasil'evskij begründete hier im 19. Jahrhundert eine wissenschaftliche Sozial- und Agrargeschichte von Byzanz. Seit dem 17. Jahrhundert erforschten die Balkanslawen (vor allem Serben und Bulgaren) die Geschichte von Byzanz wegen ihrer engen Verknüpfung mit der eigenen Geschichte. Den Beziehungen zwischen Byzanz und den Turkvölkern Innerasiens einschließlich der Ungarn widmete Gyula Moravcsik sein Lebenswerk. Die Griechen des 19. Jahrhunderts versuchten in ihrer Historiographie Byzanz als Vorläufer des modernen griechischen Staates zu erweisen.

Ein Nachklang der Geringschätzung von Byzanz aus der Sicht der klassischen Altertumskunde und gleichsam ein Akt des Vergessens war das bis zur Mitte des 20. Jahrhunderts, abgesehen von wenigen Ausnahmen, andauernde Versäumnis, kritische Editionen der byzantinischen Geschichtsquellen bereitzustellen. Erst die Gründung des CFHB hat hier eine Wende gebracht. Das erste Handbuch der byzantinischen Literatur, welches auch das lange verachtete und fast vergessene Schrifttum in der Volkssprache berücksichtigte, entstand erst spät im 19. Jahrhundert. Um diese Zeit wurde auch die erste umfassende Fachbibliographie begründet. Die systematische Erforschung der byzantinischen Urkunden setzte erst mit dem 20. Jahrhundert ein.

Seit dem frühen 19. Jahrhundert wurde allmählich die Bedeutung der byzantinischen Kunst entdeckt, aber ihre Erforschung entwickelte sich erst gegen Ende des 19. Jahrhunderts zu einer eigenständigen Disziplin. Von der dominierenden Klassischen Archäologie lösten sich erst im 20. Jahrhundert eine 
spätantike bzw. „christliche“ und zuletzt eine byzantinische Archäologie als selbständige Fächer.

Als weitgehend neue Forschungsfelder zeichneten sich im 20. Jahrhundert byzantinische Geographie, Prosopographie, Realienkunde und Lexikographie ab. Eine erste umfassende Synthese der Wirtschaftsgeschichte erschien zu Anfang des 21. Jahrhunderts.

Trotz so vieler im Laufe der Zeit entdeckten Arbeitsfelder harrt noch mancher Teilaspekt der Byzantinistik der Entdeckung oder intensiveren Bearbeitung, wie z. B. die Epigraphik. ${ }^{84}$ Aber es ginge über den Rahmen meines Referates hinaus, dem Rückblick nun noch einen Ausblick hinzuzufügen.

\section{Literaturverzeichnis}

Anastos, Milton, 1992: „Dumbarton Oaks and Byzantine Studies. A Personal Account", in: Laiou, Angeliki E. / Maguire, Henry (Hrsg.): Byzantium. A World Civilization. Washington D.C. 1992, 5-18.

Baltaeu, J., 1967: Dictionnaire de Biographie Française, Bd. 11, Paris.

Beck, Hans-Georg, 1959: Kirche und theologische Literatur im byzantinischen Reich. München, 7-23 (Entwicklung der theologischen Byzantinistik).

Beck, Hans-Georg, 1984: Der Vater der deutschen Byzantinistik. Das Leben des Hieronymus Wolf von ihm selbst erzählt. München.

Benakis, Linos G., 2002: Texts and Studies on Byzantine Philosophy. Athen.

Beševliev, Veselin, 1963: Die protobulgarischen Inschriften. Berlin.

Beševliev, Veselin, 1964: Spätgriechische und spätrömische Inschriften aus Bulgarien. Berlin.

Büttner-Wobst, Theodor, 1892: „Studien zur Textgeschichte des Zonaras“, in: Byzantinische Zeitschrift 1(1892), 202-244.

Cormack, Robin / Jeffreys, Elizabeth (Hrsg.), 2000: Through the Looking Glass. Byzantium through British Eyes. Aldershot (Referate Symposium 1995).

Darrouzès, Jean, 1981: Notitiae episcopatuum Ecclesiae Constantinopolitanae. Paris.

Der neue Pauly, Enzyklopädie der Antike, Bd. 13, Rezeptions- und Wissenschaftsgeschichte, A-Fo. Stuttgart, Weimar 1999.

Dieten, Ioannes Aloysius van (Hrsg.), 1975: Nicetae Choniatae Historia, Bd. I-II (Corpus Fontium Historiae Byzantinae 11). Berlin, New York.

Dieten, Jan-Louis van, 1975a: Entstehung und Überlieferung der Historia Rhomaike des Nikephoros Gregoras. Diss., Köln.

Dölger, Franz (Hrsg.), 1924-1965: Regesten der Kaiserurkunden des oströmischen Reiches, Teil 1-5, 1924-1965. (In aktualisierter Neuauflage erschienen danach Teil 1/2. Halbband (2003) sowie Teil 2 (1995) und Teil 3 (1977)).

Dölger, Franz / Karayannopulos, Johannes, 1968: Byzantinische Urkundenlehre. Erster Abschnitt: Die Kaiserurkunden. München.

84 S. o., Text mit Anm. 44. Siehe aber neuestens die Arbeiten von Andreas Rhoby, Wien. 
Dujčev, Ivan, 1966: „Les études byzantines chez les Slaves méridionaux et occidentaux depuis le XVII "è̀̀cle“", in: Jahrbuch der Österreichischen Byzantinischen Gesellschaft 15(1966), 73-88.

Easterling, Patricia, 2000: „From Britain to Byzantium. The study of Greek manuscripts", in: Cormack / Jeffreys, 107-120.

Effenberger, Arne, 1990: Goethe und die „Russischen Heiligenbilder“. Anfänge byzantinischer Kunstgeschichte in Deutschland. Mainz.

Eideneier, Hans (Hrsg.), 1994: Graeca recentiora in Germania. Deutsch-griechische Kulturbeziehungen vom 15. bis 19. Jh. Wiesbaden.

Eideneier, Hans, 1994a: „Martin Crusius Neograecus und die Folgen“, in: Eideneier 1994, 123-136.

Failler, Albert, 1995: „Le centenaire de l'Institut Byzantin des Assomptionnistes“, in: Revue des Études Byzantines 53(1995), 5-40.

Fögen, Marie Theres, 1994: „Johannes Löwenklau“, in: Rechtshistorisches Journal 13(1994), 197-201.

Gamillscheg, Ernst / Harlfinger, Dieter, 1981-1997 (und, ab Teil 3, 1997, Eleuteri, Paolo): Repertorium der griechischen Kopisten 800-1600. Wien, (Teil 1: Großbritannien, T. 2: Frankreich, T. 3: Rom mit dem Vatikan).

Grumel, Venance / Laurent, Vitalien / Darrouzès, Jean (Hrsg.), 1972-91: Les regestes des actes du patriarcat de Constantinople. I. Les actes des patriarches, 6 Faszikel, Paris.

Husner, Fritz, 1949: „Die Editio princeps des ,Corpus Historiae Byzantinae‘. Johannes Oporin, Hieronymus Wolf und die Fugger", in: Festschrift Karl Schwarber. Basel, 143-162.

Janin, Raymond, 1964: Constantinople byzantine. Paris ${ }^{2} 1964$.

Janin, Raymond 1969: Les églises et les monastères [de Constantinople]. Paris ${ }^{2} 1969$.

Janin, Raymond, 1975: Les églises et les monastères des grands centres byzantins. Paris.

Koder, Johannes, 2000: „Herbert Hunger (1914-2000)“, in: Jahrbuch der Österreichischen Byzantinistik 50(2000), V-XIII.

Kresten, Otto / Müller, Andreas E. 1993/94: „Die Auslandsschreiben der byzantinischen Kaiser des 11. und 12. Jahrhunderts: Specimen einer kritischen Ausgabe“, in: Byzantinische Zeitschrift 86/87 (1993/94), 402-429.

Kresten, Otto, 2000: „Herbert Hunger, Nachruf“, in: Almanach der Österreichischen Akademie der Wissenschaften 150 (2000), 457-477.

Krumbacher, Karl, 1886: Griechische Reise. Blätter aus dem Tagebuche einer Reise in Griechenland und in der Türkei. Berlin 1886. Reprint: Athen 1979.

Kukules, Phaidon, 1948-55: Byzantinón bíos kai politismós (Alltagsleben der Byzantiner). Athen.

Kurbatov, Georgij L., 1975: Istorija Vizantii (Istoriografija) [Geschichte von Byzanz (Historiographie)]. Leningrad.

Laiou, Angeliki E. (ed.), 2002: The Economic History of Byzantium. From the seventh through the fifteenth century, 1-3. Washington, D. C.

Lemerle, Paul, 1958: „Esquisse pour une histoire agraire de Byzance“, in: Revue historique 219 und 220. 
Lilie, Ralph-Johannes et al., 1998: Prosopographie der mittelbyzantinischen Zeit. Erste Abteilung (641-867). Prolegomena. (Nach Vorarbeiten F. Winkelmanns). Berlin, New York.

Litavrin, Gennadij G., 1994: „Vasilij Grigor'evič Vasil'evskij - osnovatel' sanktpeterburgskogo centra vizantinovedenija“ [Gründer des Sankt-Petersburger Zentrums der Byzantinistik] (1838-1899), in: Vizantijskij Vremennik/Byzantina Chronika 55(80)(1994), 5-21.

Mazal, Otto, 1989: Handbuch der Byzantinistik. Graz.

Moravcsik, Gyula, 1958: Byzantinoturcica, Bd. 1: Die byzantinischen Quellen der Geschichte der Türkvölker, Bd. 2: Sprachreste der Türkvölker in den byzantinischen Quellen, ${ }^{2}$ Berlin.

Moravcsik, Gyula, 1976: Einführung in die Byzantinologie. (Aus dem Ungarischen übersetzt von Engl, Géza). Darmstadt.

Müller, Andreas E., 2004: „Vom Verschwinden einer unbekannten Größe: der Byzantinist Paul Marc“, in: Hörandner, Wolfram / Koder, Johannes / Stassinopoulou, Maria A. (Hrsg.): Wiener Byzantinistik und Neogräzistik. Beiträge zum Symposion Vierzig Jahre Institut für Byzantinistik und Neogräzistik der Universität Wien im Gedenken an Herbert Hunger. Wien, 308-314.

ODB: Kazhdan, Alexander P. / Talbot, Alice-Mary et al. (Hrsg.) 1991: Oxford Dictionary of Byzantium, Bd. 1-3 (Seiten durchgezählt). New York, Oxford.

Osler, Douglas J., 1994: „Homer dethroned“, in: Rechtshistorisches Journal 13(1994), 202-218.

Ostrogorsky, Georg, 1963: Geschichte des byzantinischen Staates. München ${ }^{3} 1963$.

Pertusi, Agostino, 1966: „Le siècle de l'érudition“, in: Jahrbuch der Österreichischen BYzantinischen Gesellschaft 15(1966), 3-25.

Reddig, Wolfgang, 1990: Reise zum Erzfeind der Christenheit. Der Humanist Hans Dernschwam in der Türkei (1553-1555). Pfaffenweiler.

Reinsch, Diether, 1994: „Edition und Rezeption byzantinischer Historiker durch deutsche Humanisten“, in: Eideneier 1994, 47-63.

Robertson, John, 1997: „Gibbon's Roman Empire as a universal monarchy: the Decline and Fall and the imperial idea in early modern Europe", in: McKitterick, Rosamond / Quinault, Roland (Hrsg.): Edward Gibbon and empire. Cambridge, 247-270.

Rochow, Ilse, 1976: „Bemerkungen zu der Leipziger Handschrift des Zeremonienbuchs des Konstantin Porphyrogennetos und zu der Ausgabe von J.J. Reiske“, in: Klio 58(1976), 193-197.

Rutgers, Victor, 1998: „Auf den Spuren eines frühchristlichen Lebensideals. Hagiographie und Archäologie im spätantiken Ägypten“, in: Mitteilungen zur Spätantiken Archäologie und Byzantinischen Kunstgeschichte 1(1998), 9-27.

Sode, Claudia / Takács, Sarolta (Hrsg.), 2001: Novum Millennium. Studies on Byzantine History and Culture Dedicated to Paul Speck. Aldershot, XI-XIX.

Spieser, Jean-Michel, 2000: „Du Cange and Byzantium“, in: Cormack / Jeffreys, 199-210.

Stolte, Bernard, 1994: „The Lion's Paws. Observations on Joannes Leunclavius (1541-1594) at work“, in: Rechtshistorisches Journal 13(1994), 219-233. 
Suchland, Klaus-Henning, 2001: Das Byzanzbild des Tübinger Philhellenen Martin Crusius (1526-1607). Diss., Würzburg.

Tinnefeld, Franz, 2002: „Karl Krumbacher und der Streit um die neugriechische Schriftsprache“, in: Beyer, H.-V. et al.: Margarite Poljakovskoj kollegi, druz'ja, ućeniki (Antićnaja drevnost' i srednie veka 33). Ekaterinburg, 294-312.

Trapp, Erich et al., 1976-1994: Prosopographie der Palaiologenzeit, 12 Faszikel. Wien.

Trapp, Erich et al., ab 1994: Lexikon zur byzantinischen Gräzität. Wien.

Trapp, Erich et al., 1995: Prosopographisches Lexikon der Palaiologenzeit, Addenda zu Faszikel 1-12, Wien. (Abkürzungsverzeichnis und Gesamtregister. 1996.)

Wamser, Ludwig (Hrsg.), 2004: Die Welt von Byzanz - Europas östliches Erbe. Glanz, Krisen und Fortleben einer tausendjährigen Kultur. Katalog Ausstellung München, Archäologische Staatssammlung.

Wendebourg, Dorothea, 1994: „,Alles Griechische macht mir Freude wie Spielzeug den Kindern'. Martin Crusius und der Übergang des Humanismus zur griechischen Landeskunde“", in: Eideneier 1994, 113-121.

Zäh, Helmut, 1992: Hieronymus Wolf. Commentariolus de vita sua. Diss. München (ungedruckt, verfügbar nur auf Mikrofiche, Schuller Microfilm Gmbh, Donauwörth 1998). 
